\title{
The Effect of Antenatal Care on Professional Assistance at Delivery in Rural India
}

\author{
Vinod Mishra $\cdot$ Robert D. Retherford
}

Received: 2 February 2006/Accepted: 19 November 2006/Published online: 7 February 2008

(C) Springer Science+Business Media B.V. 2008

\begin{abstract}
Delivering births in a medical institution or at home with professional medical assistance has been shown to promote safe motherhood and child survival. Yet three-quarters of births in rural India continue to take place at home, most of them without the assistance of any trained health worker. This study examines the role of antenatal care (ANC) in promoting professional assistance at delivery, using data from India's 1992-93 and 1998-99 National Family Health Surveys (NFHS-1 and NFHS-2). We estimate the effect of number of antenatal care visits $(0,1-2,3+)$ on professional assistance at delivery (no assistance, professional assistance at home, delivery in a medical institution), using multinomial logistic regression, controlling for demographic, geographic, and socioeconomic factors, pregnancy complications, and two measures of access to health facilities. The results indicate that, after controlling for other variables (including ANC), pregnancy complications and access to health facilities do not have much effect on assistance at delivery. By contrast, ANC has a large effect, even after all other variables are controlled. The effect of ANC on professional assistance at delivery is larger in South India than in North India, and predicted percentages receiving professional assistance are higher in South India than in North India. A policy implication is that increased antenatal care coverage can be an effective means of increasing professional assistance at delivery, especially delivery in a medical institution.
\end{abstract}

Keywords Safe motherhood - Antenatal care - Skilled assistance at delivery · Reproductive health $\cdot$ Women

\footnotetext{
V. Mishra $(\bowtie)$

Demographic and Health Research Division, Macro International Inc., 11785 Beltsville Drive,

Calverton, MD, USA

e-mail: vinod.mishra@macrointernational.com

V. Mishra · R. D. Retherford

Population and Health Studies, East-West Center, Honolulu, HI, USA
} 
It is well established that giving birth under the care and supervision of trained health-care providers promotes child survival and reduces the risk of maternal mortality (Tsui et al. 1997; WHO 2004a, 2005). Maternal and child health programs in India have been promoting availability and access to trained midwives and upgrading rural health services to include facilities for institutional delivery (MOHFW 2005). Yet three-quarters of births in rural India continue to take place at home, most of them without the assistance of any trained health worker (IIPS and ORC Macro 2000). At the same time, both child mortality (especially neonatal mortality) and maternal mortality remain high. Seven out of every 100 children born in India die before reaching age one (Dyson et al. 2004), and approximately five out of every 1,000 women who become pregnant die of causes related to pregnancy and childbirth (MOHFW 2005). India accounts for more than one-fourth of all maternal deaths from causes related to pregnancy and childbirth worldwide (WHO 2004b).

Historically, improving access to services has been a primary strategy for increasing health-service utilization in developing countries. Several studies have stressed the importance of access to health services as a factor affecting the utilization of services (Rao and Richard 1984; Sarita and Tuominen 1993; Kumar et al. 1997; Rohde and Viswanathan 1995). In recent years, field experience and data from both qualitative and quantitative studies have indicated that improvements in the quality of services can further increase service utilization. Programs that maximize quality as well as access to services enhance client satisfaction, leading to greater utilization (Koenig and Khan 1999; Shelton and Davis 1996; Levine et al. 1992). It is argued that access helps determine whether an individual makes contact with the provider, while quality of care influences a client's decision whether to accept and use the service or to continue using the service (Bertrand et al. 1995). In addition to expanding health-care facilities and infrastructure, India's family welfare program has been emphasizing outreach programs, including home visits, mobile clinics, and community-based delivery systems, as mechanisms to increase both the quantity and quality of services (MOHFW 2005).

Utilization of health services is affected not only by access but also by demand for services, which is determined largely by socioeconomic factors, personal health beliefs, and perceptions of illness. A number of studies have assessed the role of socioeconomic and demographic factors in influencing demand for and utilization of maternal and child health services (Kanitkar and Sinha 1989; Elo 1992; Swenson et al. 1993; Abdalla 1993; Govindasamy 2000; Khan et al. 1994; Barlow and Diop 1995; Ahmed and Mosley 1997; Regmi and Manandhar 1997; Govindasamy and Ramesh 1997; Das et al. 2001). Of course, availability and quality of services can also influence demand for services.

In this paper, we examine factors associated with likelihood of receiving professional assistance at delivery for rural Indian women. More specifically, we examine the role of antenatal care (ANC) in promoting professional assistance at delivery. An earlier study in four Indian states observed that women who received any antenatal care were significantly more likely to deliver in a medical institution (Sugathan et al. 2001). The present study builds on this previous research to include professional assistance for noninstitutional deliveries, and expands its coverage to 
India as a whole and the North and South regions separately, using data from the 1992-93 and 1998-99 National Family Health Surveys (NFHS-1 and NFHS-2).

Antenatal care is hypothesized to have a positive effect on the likelihood of receiving professional assistance at delivery, inasmuch as women receiving antenatal care come in contact with health-care providers who are likely to encourage them to seek professional assistance at delivery or to give birth in a medical facility. A complicating factor is that women with pregnancy complications are more likely than other pregnant women both to receive antenatal check-ups and to receive professional medical assistance at delivery because of the pregnancy complication. The study examines the association between ANC and professional assistance at delivery after adjusting for the potential confounding effect of pregnancy complications.

\section{Data and Methods}

Data are from India's first and second National Family Health Surveys: NFHS-1 (conducted in 1992-93) and NFHS-2 (conducted in 1998-99). Both surveys were designed along the lines of the Demographic and Health Surveys (DHS) that have been conducted worldwide in many developing countries since the 1980s. NFHS-1 comprises 88,562 households and 89,777 ever-married women, and NFHS-2 comprises 91,196 households and 89,199 ever-married women. Details of sample design are contained in the basic survey reports for NFHS-1 and NFHS-2 (IIPS 1995; IIPS and ORC Macro 2000).

The units of analysis in our study are births to rural women that took place during the three-year period immediately preceding each survey. The analysis is confined to rural women partly because the vast majority (about 70\%) of India's population lives in rural areas, and partly because our health care access variables are meaningful only in a rural context. The analysis for rural India (all states) is based on 29,069 births that occurred during the three-year period before NFHS-1 and 25,499 births that occurred during the three-year period before NFHS-2. In order to capture some of India's demographic diversity, we also examine births to rural women in two subsets of states, one set from North India (Bihar, Uttar Pradesh, Rajasthan, and Madhya Pradesh) and one set from South India (Andhra Pradesh, Goa, Karnataka, Kerala, and Tamil Nadu).

The method of analysis is multinomial logistic regression (m-logit for short). The response variable is a three-category variable representing professional assistance at delivery (no assistance, professional assistance at home, institutional delivery). The principal predictor variable is number of antenatal care (ANC) visits (none, 1-2, $3+$ ). Other predictor variables of particular interest (because they are important variables from the point of view of the health system) are pregnancy complications (none, mild/moderate, severe) and two health care access variables: availability of a hospital within $5 \mathrm{~km}$ (no, yes), and whether the mother's village is accessible by means of an all-weather road (no, yes). Additional control variables are mother's age at childbirth in years $(15-19,20-24,25-29,30-49)$, birth order $(1,2,3,4+)$, religion of household head (Hindu, Muslim, other), caste/tribe of household head 
(scheduled caste/scheduled tribe, other), geographic region (north, central and west, east and northeast, south-not included in the models when the North and South groups of states are examined separately), mother's education (illiterate, literate but not completed middle school, middle complete or higher), mother's current work status (not working, working), mother's media exposure (not exposed, exposed), and household standard of living (low, medium, high).

Some of the predictor variables require further explanation. In the categorization of mother's education, "middle complete" is defined as eight or more completed years of education, and "less than middle complete" does not include illiterate. Scheduled castes and scheduled tribes are castes and tribes identified by the Government of India as socially and economically backward and in need of special protection from social injustice and exploitation. ${ }^{1}$ Regarding media exposure, NFHS-1 asked questions about exposure to radio (at least once a week), television (at least once a week), and cinema (at least once a month), and NFHS-2 asked not only about these three media but also about reading newspapers and magazines (at least once a week). In our analysis, low media exposure is defined as positive responses on none or one of these four types of exposure, and high media exposure is defined as positive responses on two, three, or four types. Standard of living is defined in terms of an index of ownership of various household goods, with index values grouped into high, medium, and low standard of living. ${ }^{2}$ The categories of the remaining predictor variables are self-explanatory.

\footnotetext{
${ }^{1}$ In NFHS-1, respondents were asked to report their caste/tribe, and their answers were compared with the official government list of scheduled castes/tribes before coding the response as scheduled caste, scheduled tribe, or "other." In NFHS-2, there was no checking against the official government list. Instead, respondents were first asked the name of their caste/tribe, if any. Then, if they belonged to a caste/tribe, they were asked whether the caste/tribe was a scheduled caste, a scheduled tribe, an "other backward caste," or none of these. Because of these differences in the way the questions were asked, the caste/tribe variable is not precisely comparable between the two surveys.

${ }^{2}$ In NFHS-1, the standard of living index was calculated by adding the following scores-house type: 4 for pucca (high-quality construction materials throughout), 2 for semi-pucca, 0 for kachha (low-quality construction materials throughout); toilet facility: 4 for own flush toilet, 2 for public or shared flush toilet or own pit toilet, 1 for shared or public pit toilet, 0 for no facility or other facility; source of lighting: 2 for electricity, 1 for kerosene, gas, or oil, 0 for other source of lighting; main fuel for cooking: 2 for electricity, liquid petroleum gas, or biogas, 1 for coal, charcoal, or kerosene, 0 for other fuel; source of drinking water: 2 for pipe, hand pump, or well in residence/yard/plot, 1 for public tap, hand pump, or well, 0 for other water source; separate room for cooking: 1 for yes, 0 for no; ownership of agricultural land: 4 for 5 acres or more, 3 for 2.0-4.9 acres, 2 for less than 2 acres or acreage not known, 0 for no agricultural land; ownership of irrigated land: 2 if household owns at least some irrigated land, 0 if no irrigated land; ownership of livestock: 2 if own livestock, 0 if not own livestock; durable goods ownership: 4 for a car or tractor, 3 each for a scooter/motorcycle or refrigerator, 2.5 for a television, 2 each for a bicycle, electric fan, radio/transistor, sewing machine, water pump, bullock cart, or thresher, 1 for a clock/watch. Index scores range from 0 to 10 for low SLI to 10.5-20 for medium SLI and 20.5-55 for high SLI.

In NFHS-2, the standard of living index was calculated by adding the following scores-house type: 4 for pucca, 2 for semi-pucca, 0 for kachha; toilet facility: 4 for own flush toilet, 2 for public or shared flush toilet or own pit toilet, 1 for shared or public pit toilet, 0 for no facility; source of lighting: 2 for electricity, 1 for kerosene, gas, or oil, 0 for other source of lighting; main fuel for cooking: 2 for electricity, liquid petroleum gas, or biogas, 1 for coal, charcoal, or kerosene, 0 for other fuel; source of drinking water: 2 for pipe, hand pump, or well in residence/yard/plot, 1 for public tap, hand pump, or well, 0 for other water source; separate room for cooking: 1 for yes, 0 for no; ownership of house: 2 for yes, 0 for no; ownership of agricultural land: 4 for 5 acres or more, 3 for 2.0-4.9 acres, 2 for less than 2
} 
Table 1 Sample distribution of births in the three years before NFHS-1 and NFHS-2 by type of assistance at delivery and selected predictor variables for rural areas of All India, North India, and South India (percent)

\begin{tabular}{|c|c|c|c|c|c|c|}
\hline \multirow[t]{2}{*}{ Characteristic } & \multicolumn{3}{|l|}{ NFHS-1 } & \multicolumn{3}{|l|}{ NFHS-2 } \\
\hline & All India & North & South & All India & North & South \\
\hline \multicolumn{7}{|l|}{ Assistance at delivery } \\
\hline No assistance & 74 & 85 & 47 & 67 & 81 & 35 \\
\hline Assistance at home & 10 & 8 & 14 & 8 & 7 & 9 \\
\hline Institutional delivery & 16 & 7 & 39 & 25 & 12 & 56 \\
\hline \multicolumn{7}{|l|}{ Antenatal care visits } \\
\hline None & 41 & 60 & 10 & 40 & 63 & 8 \\
\hline $1-2$ & 22 & 22 & 12 & 23 & 22 & 12 \\
\hline $3+$ & 37 & 19 & 78 & 37 & 15 & 80 \\
\hline \multicolumn{7}{|l|}{ Pregnancy complications } \\
\hline None & NA & NA & NA & 37 & 33 & 46 \\
\hline Mild/moderate & NA & NA & NA & 28 & 27 & 28 \\
\hline Severe & NA & NA & NA & 35 & 40 & 26 \\
\hline \multicolumn{7}{|c|}{ Availability of a hospital within $5 \mathrm{~km}$} \\
\hline No & 61 & 69 & 50 & 58 & 61 & 43 \\
\hline Yes & 39 & 31 & 50 & 42 & 39 & 57 \\
\hline \multicolumn{7}{|c|}{ Village has all-weather road } \\
\hline No & 51 & 59 & 40 & 58 & 66 & 46 \\
\hline Yes & 49 & 41 & 60 & 42 & 34 & 54 \\
\hline Number of births & 29,069 & 14,549 & 4,979 & 25,499 & 13,015 & 4,485 \\
\hline
\end{tabular}

NA: Not available

Note: North India includes Bihar, Uttar Pradesh, Rajasthan, and Madhya Pradesh. South India includes Andhra Pradesh, Goa, Karnataka, Kerala, and Tamil Nadu

\section{Results}

Table 1 shows the sample distribution of births on the main predictor variables. In the case of rural India as a whole, mothers of two-fifths of the births in each of the two surveys received no antenatal care (ANC) visits, and mothers of only $37 \%$ of the births received the recommended three or more ANC visits. Mothers of $35 \%$ of births in NFHS-2 reported at least one severe pregnancy complication, and mothers

Footnote 2 continued

acres or acreage not known, 0 for no agricultural land; ownership of irrigated land: 2 if household owns at least some irrigated land, 0 for no irrigated land; ownership of livestock: 2 if own livestock, 0 if not own livestock; durable goods ownership: 4 for a car or tractor, 3 each for a moped/scooter/motorcycle, telephone, refrigerator, or color television, 2 each for a bicycle, electric fan, radio/transistor, sewing machine, black and white television, water pump, bullock cart, or thresher, 1 each for a mattress, pressure cooker, chair, cot/bed, table, or clock/watch. Index scores range from 0 to 14 for low SLI to 15-24 for medium SLI to 25-67 for high SLI.

Because the standard of living index is calculated slightly differently in NFHS-1 and NFHS-2, comparisons on this index between NFHS-1 and NFHS-2 are not exact. 
of another $28 \%$ of the births reported a mild or moderate pregnancy complication. About three-fifths of the births in each of the two surveys occurred in villages within $5 \mathrm{~km}$ of a hospital. About one-half (in NFHS-1) to three-fifths (in NFHS-2) of the births occurred in villages accessible by an all-weather road. The distributions for North and South separately indicate that the South is considerably more advanced than the North on the health and development indicators shown in the table.

Table 2 shows unadjusted and adjusted effects of antenatal care on assistance at delivery for rural India as a whole. Effects are measured by relative risk ratios (RRRs) calculated for each survey separately from the fitted m-logit regression underlying each of the five models. "Unadjusted" means that effects are estimated without controlling for other variables, and "adjusted" means that effects are estimated with controls for other variables. Control variables are introduced simply by adding them to the set of predictor variables. In the unadjusted case, ANC is the only predictor variable.

In the context of Table 2, relative risks may be denoted as P1/P3 and P2/P3, where P1 denotes the predicted probability of delivery assistance at home, P2 denotes the predicted probability of institutional delivery, and P3 denotes the predicted probability of no assistance. "Predicted" means predicted from a fitted mlogit model. "No assistance" is designated (arbitrarily) as the reference category of the delivery assistance variable. In each of the five models shown in Table 2, the column labeled "assistance at home" shows RRRs based on values of P1/P3, and the column labeled "institutional delivery" shows RRRs based on values of P2/P3. In Model 1 for "assistance at home" for NFHS-2, the RRR of 2.24 is the ratio of the estimated value of P1/P3 for 1-2 ANC visits to the estimated value of P1/P3 for no ANC visits, and the RRR of 4.79 is the ratio of the estimated value of P1/P3 for 3+ ANC visits to the estimated value of P1/P3 for no ANC visits. The RRRs shown in the other columns have a similar interpretation, except that $\mathrm{P} 2 / \mathrm{P} 3$ replaces $\mathrm{P} 1 / \mathrm{P} 3$ in the case of columns pertaining to institutional delivery.

The relative risks P1/P3 and P2/P3 are sometimes referred to as "improper odds" (Retherford and Choe 1993). The adjective "improper" applies when the response variable has three or more categories, as in Table 2. In this case, the numerator and denominator of the relative risk do not add to one, as they do when the response variable has only two categories (in which case the m-logit model reduces to the ordinary logit model). This "improper" aspect of relative risks makes RRRs somewhat difficult to interpret. Consider again the RRR of 2.24 in the first column of Table 2. Because this RRR is greater than one, it suggests that P1, the probability of delivery assistance at home, is higher in the case of 1-2 ANC visits than in the case of no ANC visits. But this is not necessarily the case, because $\mathrm{P} 1$ can decrease even when $\mathrm{P} 1 / \mathrm{P} 3$ increases. This can occur if both $\mathrm{P} 1$ and $\mathrm{P} 3$ decrease as the number of ANC visits increases, and the proportionate decrease in $\mathrm{P} 3$ exceeds the proportionate decrease in $\mathrm{P} 1$. This movement in opposite directions is not possible in ordinary logit regression, where the relative risk simplifies to the (proper) odds $\mathrm{P} /(1-\mathrm{P})$, because in this case it is not possible for both numerator and denominator to decrease simultaneously; i.e., if $\mathrm{P} /$ (1-P) increases, $\mathrm{P}$ must increase and (1-P) must decrease.

Because of this ambiguity in the interpretation of RRRs, we also present values of P1, P2, and P3 as an alternative way of presenting the m-logit results. These 


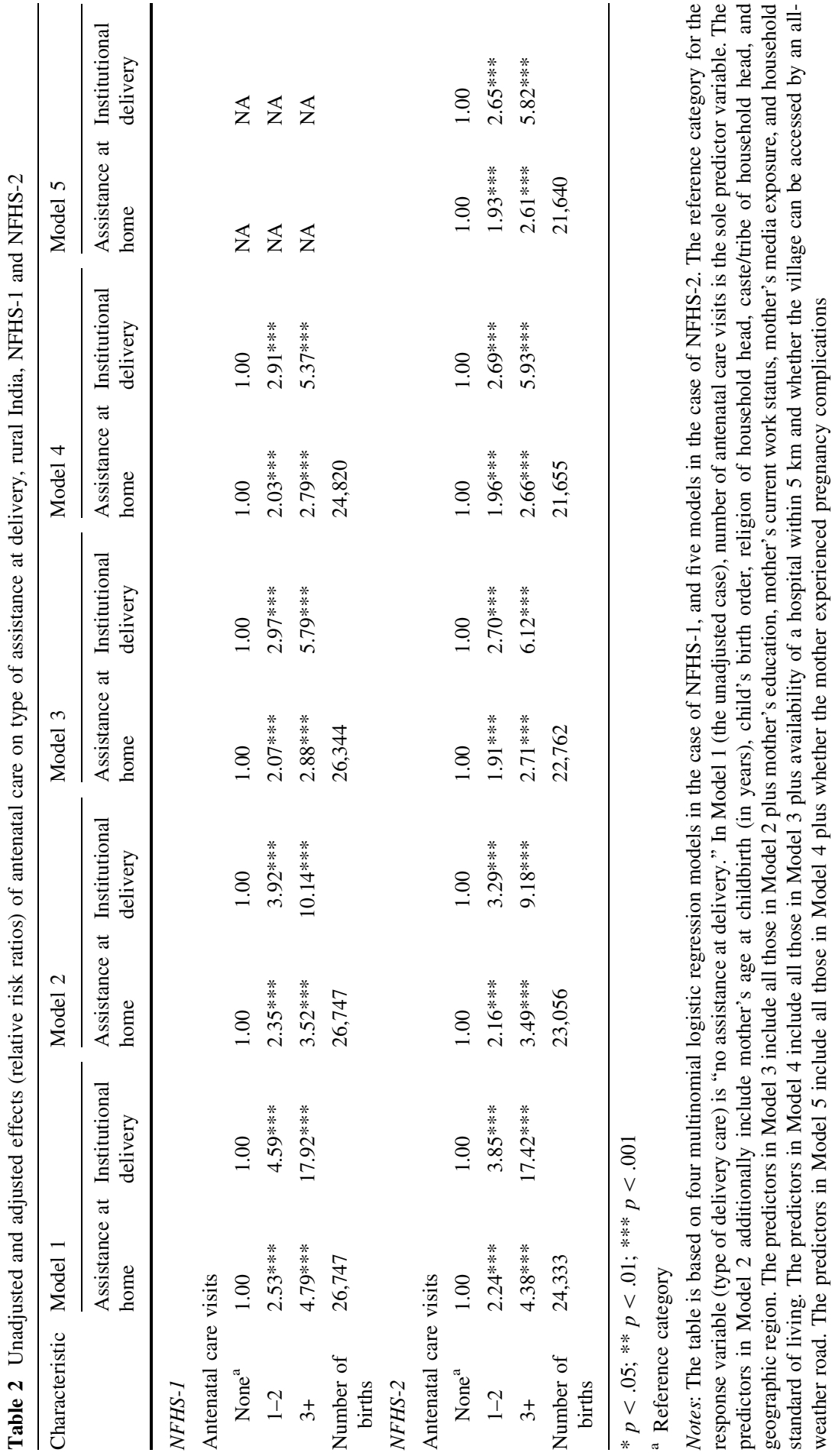


predicted probabilities are presented in the form of predicted percentages born with no assistance, assistance at home, or institutional delivery, as shown in Fig. 1 for two of the models in Table 2. When calculating the predicted percentages, it is necessary to assign values to any predictor variables other than ANC that are included in the m-logit regression, because the predicted percentages are affected by the values of the other predictors. Typically the other predictors are held constant at their mean values in the subsample of births for which the m-logit regression is run, and that is what is done here. Both modes of presentation of results-RRRs and predicted percentages - are useful. In this regard, it should be noted that RRRs have the advantage of not depending on the values of the other predictor variables.

The RRRs shown in Table 2 are rather similar for NFHS- 1 and NFHS-2, so the discussion here is limited to NFHS-2. Model 1 shows unadjusted effects of ANC on delivery assistance. RRRs rise substantially for both home assistance and institutional delivery as the number of ANC visits increases, but more so for institutional delivery than for home assistance. The RRR is especially high in the case of institutional delivery for $3+$ ANC visits.

Models 2-5 progressively add controls, as explained in detail in the footnote to Table 2. The addition of controls reduces the RRRs for assistance at home, but not greatly so. The reduction is greater in the case of institutional delivery. In Models 4 and 5, it is noteworthy that the addition of the two health care access variables (availability of a hospital within $5 \mathrm{~km}$ and accessibility of the village by an allweather road) and the pregnancy-complication variable makes little difference in the RRRs once controls have been introduced for the demographic, geographic, and socioeconomic factors. The unimportance of the controls for health care accessibility suggests that India's network of health services is extensive enough that access to ANC and delivery services is not a major problem. The unimportance of pregnancy complications indicates that this variable is not confounding the relationship between ANC and professional assistance at delivery, at least not to any significant extent. (Confounding is possible because pregnancy complications are a common cause of both ANC and professional assistance at delivery.)

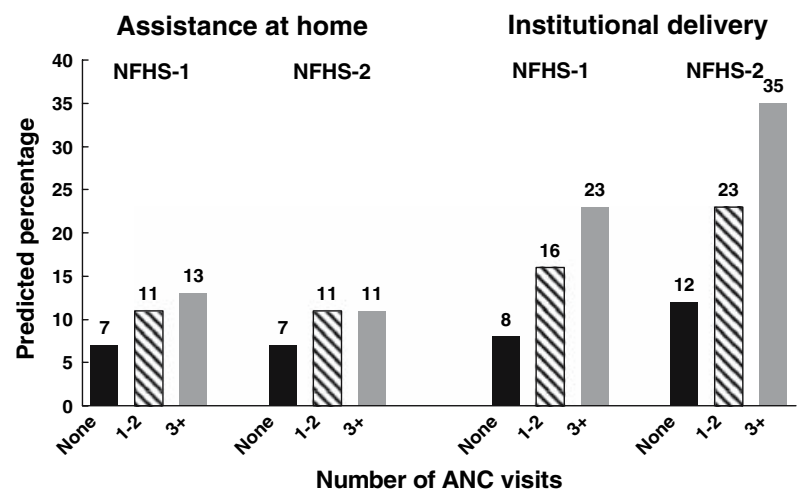

Fig. 1 Adjusted effects of antenatal care on type of assistance at delivery in rural India, NFHS-1 and NFHS-2. Source: Derived from Model 4 for NFHS-1 and Model 5 for NFHS-2 in Table 2 using the PREDICT command in STATA 


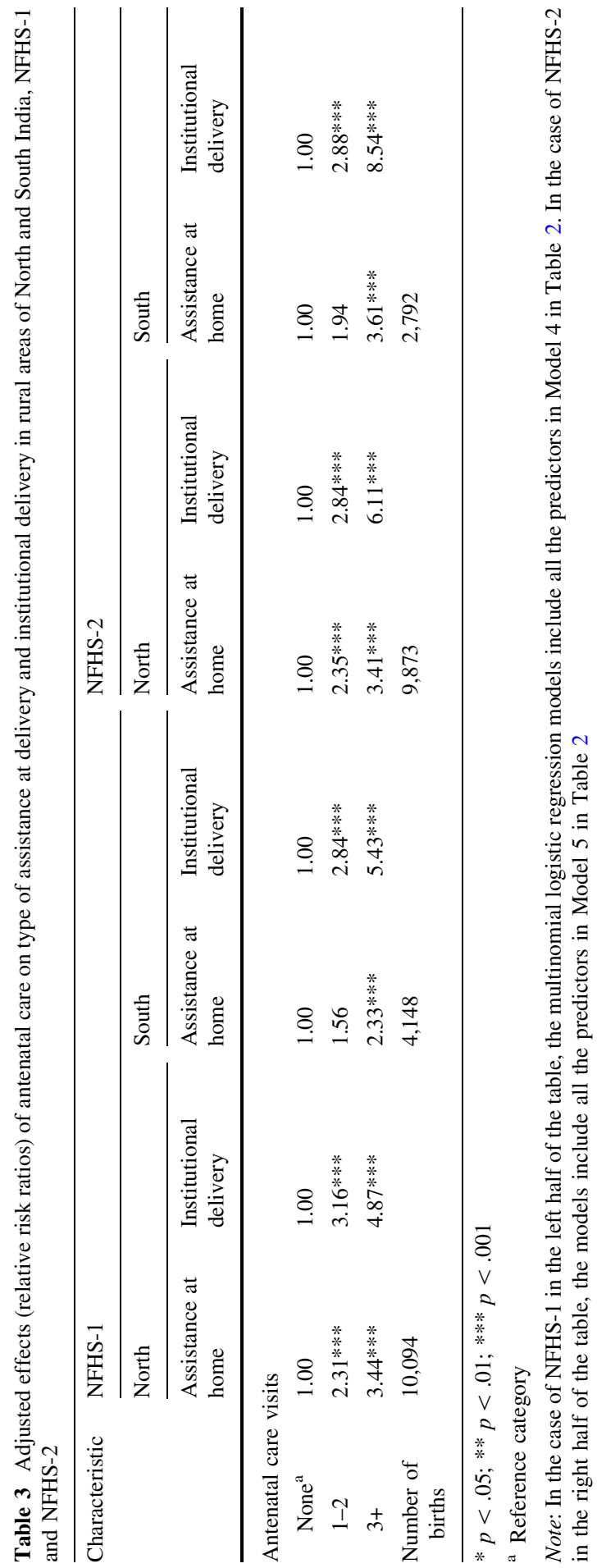


Figure 1 shows the alternative way of presenting the m-logit results, in terms of predicted percentages receiving no assistance, assistance at home, or institutional delivery by number of ANC visits. This is done only for the full models, namely Model 4 for NFHS-1 and Model 5 for NFHS-2. The predicted percentage receiving assistance at home increases much less dramatically than does the RRR as the number of ANC visits increases. This is especially true in NFHS-2, where the RRR increases from 1.93 to 2.61 between 1-2 and 3+ ANC visits, whereas P2, the predicted percentage receiving assistance at home, remains constant at $11 \%$ for both 1-2 and 3+ ANC visits. In the case of institutional delivery, both the RRR and the predicted percentage receiving institutional delivery increase dramatically as the number of ANC visits increases.

Table 3 and Figs. 2 and 3 show results for North and South separately. North and South do not differ much regarding the effect of ANC on assistance at home. By contrast, the effect of ANC on institutional delivery is larger in the South than in the

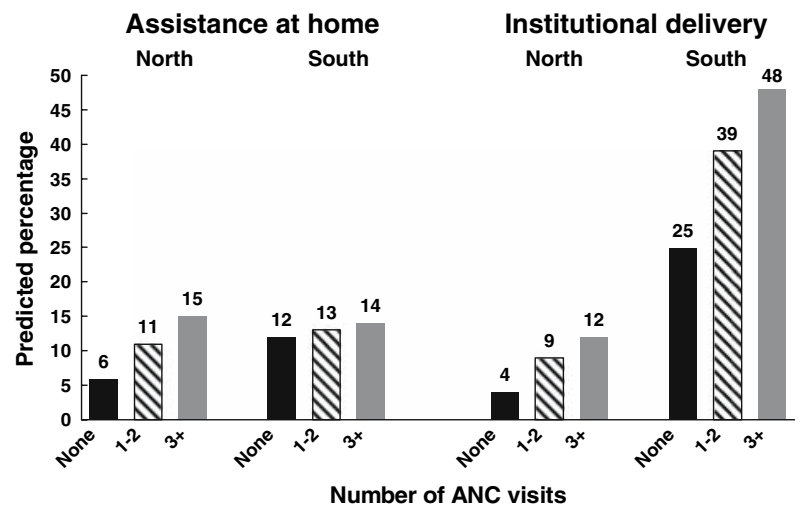

Fig. 2 Adjusted effects of antenatal care on type of assistance at delivery in rural areas of North and South India, NFHS-1. Source: Derived from the multinomial logistic regression models underlying Table 3 using the PREDICT command in STATA

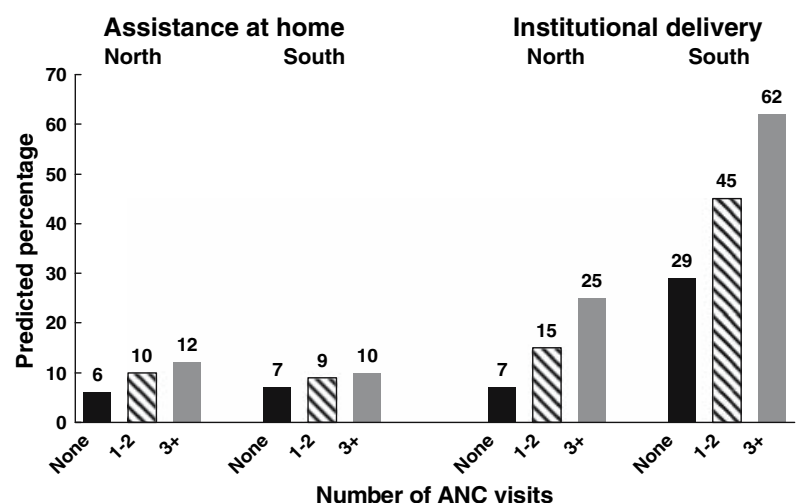

Fig. 3 Adjusted effects of antenatal care on type of assistance at delivery in rural areas of North and South India, NFHS-2. Source: Derived from the multinomial logistic regression models underlying Table 3 using the PREDICT command in STATA 
North, and the predicted percentages receiving institutional delivery are higher in the South than in the North. It is also noteworthy that in the South, the increase in RRR, but not in the predicted percentage, is especially large between 1-2 and 3+ ANC visits-again illustrating the methodological point, made earlier, that the pattern of variation in the RRR does not necessarily parallel the pattern of variation in the predicted percentage.

Table 4 and Figs. 4 and 5 show adjusted effects of pregnancy complications and the two health care access variables on assistance at delivery, based on Model 5 for NFHS-2 in Table 2, which controls for all of the other predictor variables (including number of ANC visits) considered in our study. The Table and Figures show that the effects of pregnancy complications and the two health care access variables are in the expected direction but small. By contrast, the effects of ANC on assistance at

Table 4 Adjusted effects (relative risk ratios) of selected predictor variables on type of assistance at delivery in rural India, NFHS-2

\begin{tabular}{|c|c|c|}
\hline Characteristic & Assistance at home & Institutional delivery \\
\hline \multicolumn{3}{|c|}{ Pregnancy complications } \\
\hline None $^{\mathrm{a}}$ & 1.00 & 1.00 \\
\hline Mild/moderate & $1.25 * *$ & $1.16^{*}$ \\
\hline Severe & $1.36^{* * * *}$ & $1.31 * * *$ \\
\hline \multicolumn{3}{|c|}{ Availability of a hospital within $5 \mathrm{~km}$} \\
\hline $\mathrm{No}^{\mathrm{a}}$ & 1.00 & 1.00 \\
\hline Yes & 1.06 & $1.31 * * *$ \\
\hline \multicolumn{3}{|c|}{ Village has all-weather road } \\
\hline $\mathrm{No}^{\mathrm{a}}$ & 1.00 & 1.00 \\
\hline Yes & $1.43 * * *$ & $1.32 * * *$ \\
\hline Number of births & 21,640 & \\
\hline
\end{tabular}

$* p<.05 ; * * p<.01 ; * * * p<.001$

${ }^{a}$ Reference category

Note: The underlying multinomial logistic regression model is Model 5 for NFHS-2 in Table 2

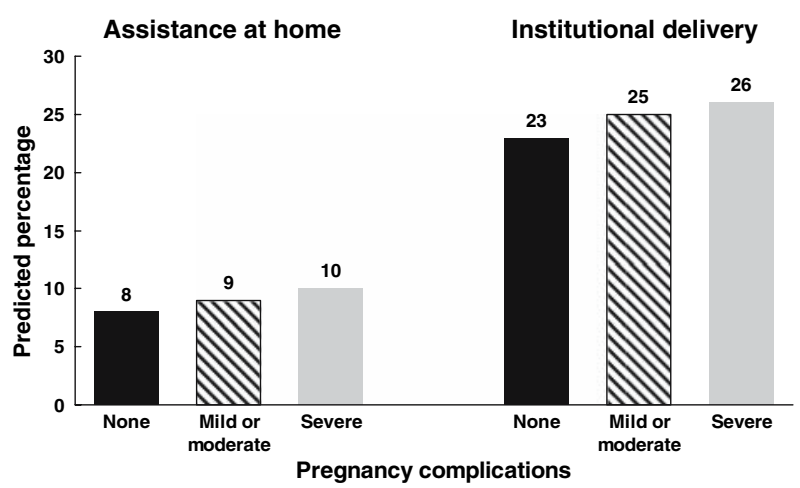

Fig. 4 Adjusted effects of pregnancy complications on type of assistance at delivery, NFHS-2. Source: Multinomial logistic regression model underlying Table 4 using the PREDICT command in STATA 


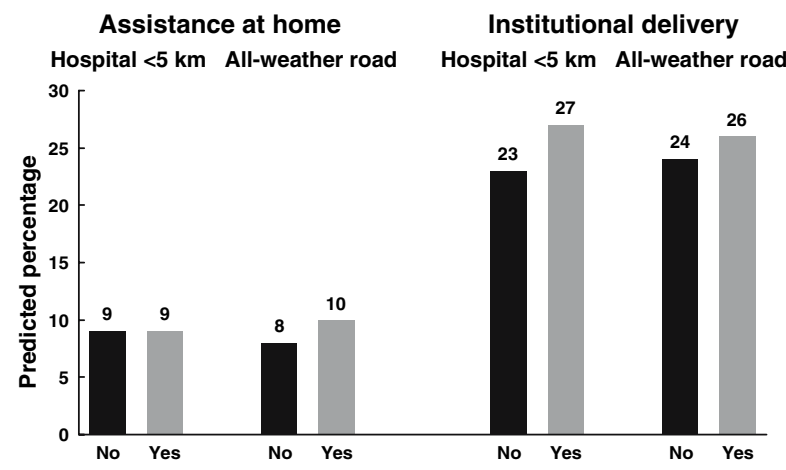

Fig. 5 Adjusted effects of health-care-access variables on type of assistance at delivery, NFHS-2. Source: Multinomial logistic regression model underlying Table 4 using the PREDICT command in STATA

delivery (derived from the same m-logit regression) are much larger, as shown earlier.

\section{Conclusion}

The analysis indicates that antenatal care has a large effect on type of professional assistance at delivery. The effect on assistance at home is comparatively small, and the effect on institutional delivery is comparatively large, even after controlling for a large number of demographic, geographic, socioeconomic, and health care access variables. The effect of ANC on institutional delivery is larger in the South than in the North, and the predicted percentages receiving institutional delivery are higher in the South than in the North. With other variables controlled, the effects of pregnancy complications and health care access variables on assistance at delivery are in the expected direction but small. By contrast, the effects of ANC on assistance at delivery are large.

Overall, the analysis indicates that improvements in antenatal care coverage are an effective means for increasing professional assistance at delivery, and especially for increasing institutional delivery. Since three out of four deliveries in rural India still occur at home, most without any professional assistance, efforts to train traditional birth attendants, increase the availability of trained midwives, and promote home visits by paramedics for antenatal care need further strengthening.

Acknowledgment The authors thank Alfredo Fort and Fred Arnold for their comments. They also thank Gayle Yamashita and Sally Dai for programming and research assistance. An earlier version of this paper was presented at the 2004 annual meeting of the American Public Health Association in Washington, DC. Partial support for this research was provided by the United States Agency for International Development (USAID) through the MEASURE Demographic and Health Surveys project (\# GPO-C-00-03-00002-00). Views presented in the paper do not represent the views of USAID or the organizations to which the authors belong. 


\section{References}

Abdalla, G. M. (1993). Determinants of maternal and child health services utilization in Egypt. In $C D C$ 23rd Annual Seminar on Population and Development Issues in the Middle East, Africa and Asia, compiled by Cairo Demographic Centre, Cairo, Egypt.

Ahmed, S., \& Mosley, W. H. (1997). Simultaneity in maternal-child health care utilization and contraceptive use: Evidence from developing countries. Hopkins Population Center Papers on Population, WP 97-03. Baltimore MD: Department of Population Dynamics, School of Public Health, Johns Hopkins University.

Barlow, R., \& Diop, F. (1995). Increasing the utilization of cost-effective health services through changes in demand. Health Policy and Planning, 10(3), 284-295.

Bertrand, J. T., Hardee, K., Magnani, R. J., \& Angle, M. A. (1995). Access, quality of care and medical barriers in family planning programs. International Family Planning Perspectives, 21(2), 64-69.

Das, N. P., Mishra, V. K., \& Saha, P. K. (2001). Does community access affect the use of health and family welfare services in rural India? National Family Health Survey Subject Reports No. 18. Mumbai: International Institute for Population Sciences; and Honolulu: East-West Center.

Dyson T., Cassen R., \& Visaria L. (Eds.). (2004). Twenty-first century India: Population, economy, human development, and the environment. New York: Oxford University Press.

Elo, I. T. (1992). Utilization of maternal health-care services in Peru: The role of women's education. Health Transition Review, 2(1), 49-69.

Govindasamy, P. (2000). Poverty, women's education and utilization of health services in Egypt. In B. García (Eds.), Women, poverty and demographic change (pp. 263-285). New York: Oxford University Press.

Govindasamy, P., \& Ramesh, B. M. (1997). Maternal education and the utilization of maternal and child health services in India. National Family Health Survey Subject Reports, No. 5. Mumbai: International Institute for Population Sciences; and Calverton: Macro International, Demographic and Health Surveys (DHS).

International Institute for Population Sciences [IIPS]. (1995). National Family Health Survey (MCH and Family Planning), India 1992-93. Bombay, India: IIPS.

International Institute for Population Sciences [IIPS] and ORC Macro. (2000). National Family Health Survey (NFHS-2), 1998-99: India. Mumbai, India: IIPS.

Kanitkar, T., \& Sinha, R. K. (1989). Antenatal care services in five states of India. In S. N. Singh, M. K. Premi, P. S. Bhatia, \& A. Bose (Eds.), Population transition in India (Vol. 2, pp. 201-211). Delhi, India: B.R. Publishing.

Khan, Z., Soomro, G. Y., \& Soomro, S. (1994). Mother's education and utilization of health care services in Pakistan. Pakistan Development Review, 33(4, Pt 2), 1155-1166.

Koenig, M. A., \& Khan, M. E. (1999). Improving quality of care in India's family welfare programme: The challenge ahead. New York: The Population Council.

Kumar, R., Singh, M. M., \& Kaur, M. (1997). Impact of health centre availability on utilization of maternity care and pregnancy outcome in a rural area of Haryana. Journal of the Indian Medical Association, 95(8), 448-450.

Levine, R. E., Cross, H. E., Chhabra, S., \& Viswanathan, H. (1992). Quality of health and family planning services in rural Uttar Pradesh: The client's views. Demography India, 21(2), 247-265.

Ministry of Health, Family Welfare [MOHFW]. (2005). Annual report, 2003-2004. New Delhi, India: MOHFW, Government of India.

Rao, P. S., \& Richard, J. (1984). Socioeconomic and demographic correlates of medical care and health practices. Journal of Biosocial Science, 16(3), 343-355.

Regmi, G. P., \& Manandhar, M. (1997). Patterns and determinants of health care utilization. In Insights on family health issues in Nepal (pp. 27-48). Compiled by Family Health Division, Department of Health Services, Ministry of Health, Nepal, and Demographic and Health Surveys (DHS), Macro International, Calverton MD.

Retherford, R. D., \& Choe, M. K. (1993). Statistical models for causal analysis. New York: John Wiley and Sons, Inc.

Rohde, J., \& Viswanathan, H. (1995). The rural private practitioner. New York: Oxford University Press.

Sarita, P. T., \& Tuominen, R. (1993). Use of health care services in two rural communities in Tanzania. Community Dentistry and Oral Epidemiology, 21(3), 133-135. 
Shelton, J. D., \& Davis, S. S. (1996). Some priorities in maximizing access to and quality of contraceptive services. Advances in Contraception, 12(3), 233-237.

Sugathan, K. S., Mishra, V., \& Retherford, R. D. (2001). Promoting institutional deliveries in rural India: The role of antenatal care services. National Family Health Survey Subject Reports, No. 20. Mumbai, India: International Institute for Population Sciences, and Honolulu HI: East-West Center.

Swenson, I. E., Thang, N. M., Nham, V. Q., \& Tieu, P. X. (1993). Factors related to the utilization of prenatal care in Vietnam. Journal of Tropical Medicine and Hygiene, 96(2), 76-85.

Tsui, A. O., Wasserheit, J. N., \& Haaga, J. G. (1997). Reproductive health in developing countries: Expanding dimensions, building solutions. Washington, DC: National Academy Press.

World Health Organization [WHO]. (2004a). Making pregnancy safer: The critical role of the skilled attendant. A joint statement by WHO, ICM and FIGO. Geneva, Switzerland: WHO.

World Health Organization [WHO]. (2004b). Maternal mortality in 2000: Estimates developed by WHO, UNICEF and UNFPA. Geneva, Switzerland: WHO.

World Health Organization [WHO]. (2005). World health report 2005: Make every mother and child count. Geneva, Switzerland: WHO. 